\title{
Sentiment Analysis of Arabic Tweets in Sudanese Dialect
}

\author{
Huda Jamal Abdelhameed, Susana Muñoz- Hernández
}

\begin{abstract}
Sentiment analysis is the field of science that deals with extracting opinions embedded in human oral or written speech. In this paper we focus on sentiment analysis of Arabic tweets written using either Modern Standard Arabic or Sudanese dialectical Arabic. We have created our own lexicon which contain 2500 words and we have applied three different classifiers on the dataset namely; Support Vector Machine (SVM), Naive Bayes (NB) and K-Nearest Neighbor (K-NN), to classify the tweets based on its polarity into positive or negative. We evaluate our work by four different measures which are Precision, Recall, Accuracy and F-measure. The results show that, SVM achieved the best Recall, Accuracy and F-measure and it equals $95.1 \%, 76.5 \%$ and $84.4 \%$ respectively. While NB achieved best Precision and it equals to $85.1 \%$.
\end{abstract}

Index Terms - Sentiment Classification, Opinion Mining Sentiment Analysis, Social Media, Sudanese Dialect, Arabic natural Language.

\section{INTRODUCTION}

Opinion mining or sentiment analysis is the field of science that is interested in extracting opinions that very frequently are embedded in customer's comments. It has been extensively studied in the literature for the English language. By comparison, relatively few works have targeted sentiment analysis in Arabic texts [2]. There are several granularities for sentiment analysis. A popular work is to determine whether a text is subjective or objective [4]. Another common work is to determine whether a text is written to express a positive or negative opinion [3]. Sentiment analysis deal with extracting the polarity of the text (positive, negative or neutral). A third category deals with finding the strength of an emotional state in text. Such as "happy", "sad" and "angry" [2]. There are two approaches for detecting sentiment in any text [18]. The first one relies on linguistic resources such as dictionaries and lexicons [5]. The second one is based on machine learning [5]. Some researchers have combined the previous two approaches. The lexicons are very hard to build manually and they are depending on the domain. Sentiment analysis is hard to detect for many reasons; one reason is that people use different writing styles to express their opinions. A second reason is that sentiment is context dependent [6]. One of the problems in Arabic is using a noun with positive polarities as a person names such as the word (Saleem); which means Right in English. Saleem as an adjective indicates positive sentiment but as a person name it is neutral (i.e. it has no sentiment). In general Arabic is divided into three types: Classical Arabic, Modern Arabic, and Colloquial Arabic. As the official language of 22 countries, there are 49 million

Huda Jamal Abdelhameed, Department of Computer Systems and Networks, College of Computer Science and Information Technology, Sudan University of Science and Technology, Khartoum, Sudan.

Susana Munoz - Hernández, Escuela Técnica Superior de Ingenieros Informáticos Campus de Montegancedo, Boadilla del Monte, Madrid, Spain
Arab users of Facebook [7]. Arabic language is a high complex language, which embeds five critical challenges for Natural Language Processing (NLP) task,1) Arabic is not a case-sensitive language; it has no capital letters. 2) Arabic is a high inflectional language; often a single word has more than one affix, such that it may be expressed as a combination of prefix(s), lemma, and suffix(s) [8]. 3) Arabic has some variants in spelling and typographic forms. 4) Arabic texts have different meanings. For example, "Ragab" in Arabic may be used as a person name, or month. 5) Arabic resources, such as corpora, gazetteers, and NLP tools, are not free [9]. Existing Facebook sentiment analysis focus on the English language but very few focuses on Arabic slang comments [7]. It is classified into many regional forms in the Middle East [10], which are Arabian Peninsula Arabic (Khaliji Arabic), Syro-Palestinian Arabic, Egyptian Arabic and Maghrebi Arabic.

On the other hand, the analysis of social media has attracted a great deal of attention recently. Because the users of social media generate a huge volume of comments on a daily basis These reviews and comments reflect the opinions of users about different issues and it is very interesting to be able to detect the positive and negative comments.

\section{RELATED WORKS}

A) Tagwa M. [31] presented some of previous works in sentiment analysis by using two techniques: a lexicon-based technique and a Corpus-based technique. They addressed some experiments and studies that deal with sentiment analysis in Arabic. Their study aims to use sentiment classification for Arabic tweets around Khartoum. They used different techniques for Arabic sentiment analysis applied in Arabic tweets around Khartoum and decide if the sentiment is happiness (positive), sadness (negative) or neutral. The methodology of their work is creating a corpus of Arabic tweets around Khartoum. Then build a lexicon for Arabic words. This lexicon contains a total of words divided in two groups; the words indicating happiness (positive) and sadness words (negative) with experts in language. They used two types of classification techniques, SVM and naive Bayes.

B) Afnan A. Al-Subaihin et al. [17] proposed a lexicon-based sentiment analysis tool for colloquial Arabic text used in chatting, daily conversation and within social media. They have an independent component in their work which is game-based lexicons, that are based on human expertise. SVM, Naïve Bayes and Maximum Entropy classifiers are used in this study. However, they have proven that, SVM achieved the higher accuracy. Their tool should rely partially 
texts based on human judgment to overcome the problem arise from using non-standardized colloquial Arabic text.

C) Taysir H. A. Soliman et al. [14] built a sentiment analysis approach for Slang Sentimental Words and Idioms Lexicon (SSWIL) of opinions. They proposed a Gaussian kernel SVM classifier for Arabic slang language to classify Arabic news's comments on Facebook. They collected 1846 comments from news websites like: Aljazera1, BBCarabic2, Alyoum Alsabe3 and Alarabia4 and Constitution Facebook Page. They applied three types of classification. The first classification type using Classical Lexicon (SVM) without SSWIL, the second type using Classic Lexicon and SSWIL, and the third type using SSWIL only. They show that the extraction techniques fail to extract the opinion words at the first classification type but it performs well at the second type after adding the SSWIL. The first classification type produces $5.35 \%$ accuracy rate, while the second classification type produce $86.86 \%$ and applying the system using SSWIL only, it gives $43.02 \%$ as a percent of comments classification and $56.98 \%$ not classified. The results are enhanced in the second type after applying SSWIL lists.

D) Al-Kabi et al. [13] built a novel sentiment analysis tool called colloquial Non-Standard Arabic - Modern Standard Arabic-Sentiment Analysis Tool (CNSAMSA- SAT), for both colloquial and modern standard Arabic MSA. They collected 1,080 Comments and reviews from 70 social media, and manually assigned each of them to one of the three polarity values (positive, negative, and neutral). The collected Arabic reviews and comments use Egyptian, Iraqi, Jordanian, Lebanese, Saudi, and Syrian dialects. A Naive Bayes classifier is used to determine the comment or review domain. The results show that, the accuracy of determining the polarity was $90 \%$ yields, with a $10 \%$ error rate. They identify some of the reasons that may show limitations in the tool such as the polarity of some of the phrases depends mainly on the domain they were used into. For example, the Arabic word (high) within the comment "This is a high cost product." leads to consider the polarity of the comment as negative, while using the same Arabic word (high) within the comment "High-quality service" leads to consider the polarity of the comment as positive. These two Arabic sample comments demonstrate why the effectiveness of the developed tool to determine the polarity of each Arabic comment within a domain is better than its effectiveness when it is applied on a general dataset.

E) Mohammed N. Al-Kabi et al. [10] developed an opinion mining and analysis tool for Arabic language (Standard or MSA and colloquial). The tool accepts comments and opinions as input. And it is capable to identify the polarity, subjectivity, and strength of each comment. They build 18 lexicons manually. Two general purpose lexicons were built to identify polarity, and 16 domain-specific lexicons were built to identify the polarity with eight different domains: Technology, Books, Education, Movies, Places, Politics, Products and Society. They used Naive Bayes to classify the domain of the comments. Their experiments showed that the proposed tool yields more accurate results when it is applied on domain-based Arabic comments relative to general- based Arabic comments. As they present the tool yield $93.9 \%$ accuracy to classify the comments into their proper domains, a
$90 \%$ accuracy to identify the real polarity, and a $96.9 \%$ accuracy to identify the strength of the comments. This study used a small dataset, and the proposed tool is incapable to deal with emoticons and chat language.

F) Al-Kabi et al. [11] collected and analyzed Arabic comments from social network (Yahoo!). They detailed analysis of different information such as the reviews' length, numbers of likes/dislikes, polarity distribution and the languages used. The total number of the Arabic reviews and comments used in this study is 4625 , contains the topic, comments, manual polarity, gender of the users. which leads to unbalanced classes 2812, 1230, and 583 for negative, positive and neutral classes, respectively. They applied two classifiers (SVM and Naïve Bayes) on these datasets and compared between them. The result illustrates that the best accuracy achieved is $68.2 \%$ using the SVM.

\section{TOOLS AND TECHNIQUES}

In our work the tool we have used is RapidMiner and we have worked with three different classification techniques.

A) RapidMiner

RapidMiner is a java-based open source data mining and machine learning software. It has a graphical user interface (GUI) where the user can design his machine learning process without having to code [16]. Then all process is transformed into an XML (extensible Markup Language) file. RapidMiner includes many operators that support text mining such as Text Processing package. It includes more operators such as tokenization, stemming and filtering stop words. The tool can deal with the Arabic language that's why we have chosen it. B)Classification Techniques

In our work we have used three different type of classification techniques which are Support Vector Machine (SVM), Naive Bayes (NB) and K-Nearest Neighbor (K-NN).

\section{Support Vector Machines classification approach:}

Support Vector Machines (SVM) is one of the discriminative classification approaches which is commonly recognized to be more accurate. SVM classification approach is based on Structural Risk Minimization (SRM) principle from statistical learning theory. SRM is an inductive principle for model selection used for learning from finite training data and it provides a method for controlling the generalization ability of learning machines that uses a small size training data. The idea of this principle is to find a hypothesis to guarantee the lowest true error. In addition to this, the derivation of SVM is mathematically rigorous and very open to theoretical understanding and analysis.

SVM needs both positive and negative training datasets which are uncommon for other classification methods. It is outstanding from the others with its better classification performance and its ability in handling documents with high-dimensional input space and culls out most of the irrelevant features. The good generalization characteristic of SVM is due to the implementation of SRM which entails finding an optimal hyper-plane, thus guaranteeing the lowest classification error. Besides, a capacity which is independent of the dimensionality of the feature space makes SVM a highly accurate classifier in most applications. However, the major drawback of SVM is its relatively complex training and categorizing algorithms and also the high time and memory consumptions during the training stage and classifying stage 
due to its convoluted training and categorizing algorithms. Besides, confusions occur during the classification tasks because the documents could be annotated to several categories because of similarities are typically calculated individually for each category [19].

\section{Naïve Bayes classification approach:}

It is a classification technique based on Bayes' Theorem with an assumption of independence among predictors. In simple terms, a Naive Bayes classifier assumes that the presence of a particular feature in a class is unrelated to the presence of any other feature. Even if these features depend on each other or upon the existence of the other features, all of these properties independently contribute to the probability. Naive Bayes model is easy to build and particularly useful for very large data sets. Along with simplicity, Naive Bayes is known to outperform even highly sophisticated classification methods [20].

\section{K-Nearest Neighbor (K-NN) classification approach:}

K-Nearest Neighbor (K-NN) is an instant-based learning algorithm that categorized objects based on closest feature space in the training set. The training data is mapped into multi-dimensional feature space. The feature space is partitioned into regions based on the category of the training set. A point in the feature space is assigned to a particular category if it is the most frequent category among the $\mathrm{k}$ nearest training data. During the classifying stage, KNN classification approach finds the $\mathrm{k}$ closest labeled training samples for an unlabeled input sample and assigns the input sample to the category that appears most frequently within the $\mathrm{k}$ subset. As KNN outperforms the other classification approaches by its simplicity, it only requires a small training set with small number of training samples, an integer which specifies the variable of $\mathrm{k}$ and a metric to measure closeness [16].

\section{EXPERIMENTATION}

Our experiment consists of the following three phases:

\section{Data collection:}

One of difficulties for Arabic language is the lack of publicly available Arabic lexicons [27] in comparison with English. And in general, for sentiment analysis, it is better to collect a large amount of data to be used for training the classifier. Because increasing the amount of training data in the dataset, it is always improving the accuracy of the classification.

In our work, we started the first step by building our own lexicon which contains 2500 words from Sudanese dialect. It is manually collected from different social media and Arabic websites. We used Twitter's API to collect Arabic tweets. The collected data has different sizes and different categories used for training and testing.

In the second step, we classified the words manually into positive and negative. We have 608 positive and 1246 negative words as a training dataset. And we used 646 tweets collected from twitter as testing dataset.

\section{Data Preprocessing:}

This phase includes subphases as the following:

- Data cleaning: removing irrelevant information, such as URLs and special characters, for example @, \&.

- Removing duplicated characters: this is a common practice in tweets and other social media in Arabic, where one of the letters is repeated many times, for example "رهيبييبي" which mean nice in English. We reduced any repeated characters in to two character.

- Tokenization: we separated out words from tweets into tokens. These tokens could be words even words of only one characters. We extracted any alphanumeric string between two white spaces.

- Removing stopword: we removed any word carry no information. By other words we removed any word that doesn't have a meaning (articles preposition), such as "which mean "in", "on", "from "in English.

- Stemming: We removed any affixes (prefixes that added to the beginning of the word, infixes that added to the middle of the word, or/and suffixes that added to the ending of the word) from words to reduce these words to their stems or roots under the assumption that words with the same stem are semantically related. For example, we reduced "scientist" and "sciences" to "science".

- Normalization: we transformed tweets into a single canonical form that it might not have had before. We eliminated the diacritical markings, non-letters, letter Hamza (s). Also, replaced I and ! with I, replaced final $ى$ with , and replaced final $\ddot{\circ}$ with $\circ$.

Table 1: Example of preprocessing a tweet

\begin{tabular}{|c|c|}
\hline Preprocessing Step & Tweets After Preprocessing \\
\hline The original tweet & أنا ارفض صفوف العيش شدييييد \\
\hline Data cleaning & أنا ارفض صفوف العيش شدييييد \\
\hline $\begin{array}{l}\text { Removing Duplicated } \\
\text { Characters }\end{array}$ & أنا ارفض صفوف العيش شدييد \\
\hline Tokenization & أنا، ارفضيد، ،دي، شفوف، البهيش، دئ، دي \\
\hline Stopword Removal & أنا ارفض صفوف العيش شدييد \\
\hline Stemming & أنا رفض صف عيش شدييد بهدل \\
\hline Normalization & انا رفض صف عيش شدييد بهدل \\
\hline
\end{tabular}

\section{Sentiment Classification:}

The data is divided into training and testing dataset. Training dataset used to build the classification models based on SVM, NB and K-NN classifiers. The data classified based on its polarity to positive and negative classes. Testing dataset is used to predict the polarity of the tweets.

Table 2: Sample of Classified Tweets.

\begin{tabular}{|c|c|c|}
\hline Tweet & In English & Class \\
\hline الفلم دا حلو حلاة & $\begin{array}{l}\text { This movie is } \\
\text { nice. }\end{array}$ & Positive \\
\hline الزول دا قلبو مقطووووع & $\begin{array}{l}\text { This is afraid } \\
\text { person }\end{array}$ & Negative \\
\hline البت دي فاكاها في شديد & $\begin{array}{l}\text { This is a } \\
\text { conceited girl }\end{array}$ & Negative \\
\hline |لاكل دا ظا||(بط & $\begin{array}{l}\text { This is } \\
\text { delicious food }\end{array}$ & Positive \\
\hline اللوشن دا طلع ماسورة & $\begin{array}{l}\text { This lotion is } \\
\text { not good }\end{array}$ & Negative \\
\hline
\end{tabular}




\section{Sentiment Analysis of Arabic Tweets in Sudanese Dialect}

\section{RESUlts}

Four different measures were calculated which are Precision, Recall, Accuracy and F-Measure for every classifier, to evaluate the correctness of classifying testing tweets as positive or negative. Table 3 below shows a confusion matrix that introduces these measures.

Table 3: Confusion Matrix for Two Classes Pos and Neg

\begin{tabular}{|l|l|l|}
\hline & \multicolumn{2}{l|}{ Predicted Class } \\
\hline Actual Class & Pos & Neg \\
\hline Pos & TP & FN \\
\hline Neg & FP & TN \\
\hline
\end{tabular}

In the table above we have four parameters (True Positive (TP), True Negative (TN), False Positive (FP) and False Negative (FN)), which are needed to calculate the measures. TP: is the number of tweets that were correctly classified as positive.

TN: is the number of tweets that were correctly classified as negative.

FP: is the number of tweets that were classified as positive but they are negative.

FN: is the number of tweets that were classified as negative but they are positive.

Therefore, the formula of the measures is the following:

Precision $=\mathrm{TP} /(\mathrm{TP}+\mathrm{FP})$

Recall $=\mathrm{TP} /(\mathrm{TP}+\mathrm{FN})$

Accuracy $=(\mathrm{TP}+\mathrm{TN}) /(\mathrm{TP}+\mathrm{FP}+\mathrm{FN}+\mathrm{TN})$

F-measure $=2 *($ Precision $*$ Recall $) /($ Precision $*$ Recall $)$

Table 4, 5, 6, 7, 8 and 9 below, shows the results of each classifier in our experiments with our dataset.

Table 4: True Positive and True Negative for the Support Vector Machine

\begin{tabular}{|l|l|l|}
\hline & TP & TN \\
\hline Predicted Positive & 234 & 61 \\
\hline $\begin{array}{l}\text { Predicted } \\
\text { Negative }\end{array}$ & 374 & 1185 \\
\hline
\end{tabular}

Table 5: Class Precision, Recall, Accuracy and F-Measure for the Support Vector Machine

\begin{tabular}{|l|l|l|l|l|}
\hline Classifier & Precision & Recall & Accuracy & F-Measure \\
\hline SVM & $\mathbf{7 6 . 0 1 \%}$ & $\mathbf{9 5 . 1 0 \%}$ & $\mathbf{7 6 . 5 \%}$ & $\mathbf{8 4 . 4 \%}$ \\
\hline
\end{tabular}

From table 5 above we notice that, Support Vector Machine achieved good results for Recall which equal to $95.10 \%$.
Table 6: True Positive and True Negative for the Naïve Bayes

\begin{tabular}{|l|l|l|}
\hline & TP & TN \\
\hline Predicted Positive & 518 & 731 \\
\hline $\begin{array}{l}\text { Predicted } \\
\text { Negative }\end{array}$ & 90 & 515 \\
\hline
\end{tabular}

Table 7: Class Precision, Recall, Accuracy and F-Measure for the Naïve Bayes

\begin{tabular}{|l|l|l|l|l|}
\hline Classifier & Precision & Recall & Accuracy & F-Measure \\
\hline SVM & $\mathbf{8 5 . 1 2} \%$ & $\mathbf{4 1 . 3 3 \%}$ & $\mathbf{5 5 . 7 1 \%}$ & $\mathbf{5 5 . 5 \%}$ \\
\hline
\end{tabular}

In table 7 above, Naïve Bayes achieved good result for Precision which equal to $85.12 \%$.

Table 8: True Positive and True Negative for the K-Nearest Neighbor

\begin{tabular}{|l|l|l|}
\hline & TP & TN \\
\hline Predicted Positive & 180 & 63 \\
\hline $\begin{array}{l}\text { Predicted } \\
\text { Negative }\end{array}$ & 428 & 1183 \\
\hline
\end{tabular}

Table 9: Class Precision, Recall, Accuracy and F-Measure for the K-Nearest Neighbor

\begin{tabular}{|l|l|l|l|l|}
\hline Classifier & Precision & Recall & Accuracy & F-Measure \\
\hline SVM & $\mathbf{7 3 . 4 3 \%}$ & $\mathbf{9 4 . 9 4 \%}$ & $\mathbf{7 3 . 5 \%}$ & $\mathbf{8 2 . 8 \%}$ \\
\hline
\end{tabular}

From table 9 above we notice that, K-Nearest Neighbor achieved good result for Recall which equal to $94.94 \%$.

Figure 1 below shows a composition of the results of the three classifiers in detail.

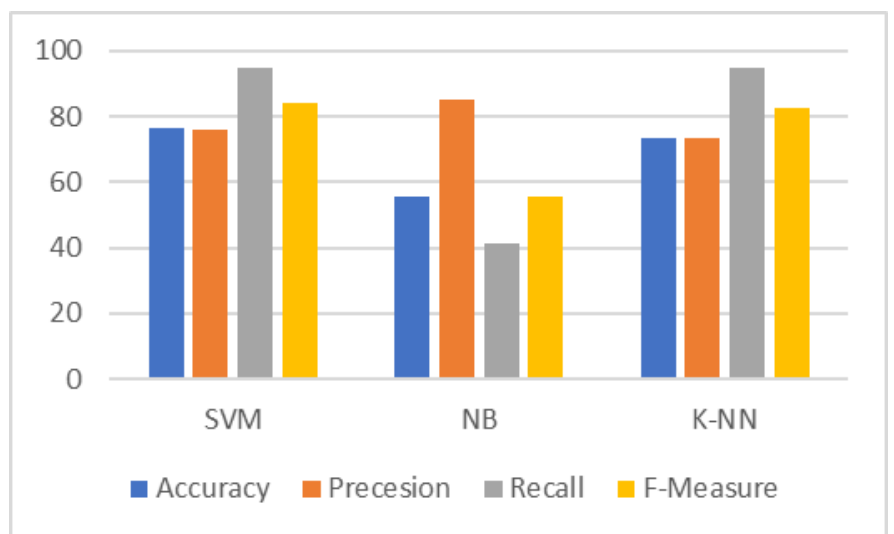

Figure 1: Accuracy, Precision, Recall and F-measure for the three classifiers.

From figure 1 above we found that the best Accuracy, Recall and F-measure was achieved by Support Vector Machine. While the best Precision was achieved by Naïve Bayes. 


\section{CONCLUSION}

This paper considered sentiment analysis in Arabic tweets which are written in Sudanese dialect. A new lexicon for Sudanese dialect was built, which consists of 2500 tweets. We split the data into training and testing sets. The SVM, Naïve Bayes and K-NN classifiers were applied to detect the polarity of the given tweets of the training set. The results of posterior experiments with the testing set show that, SVM achieved the best Accuracy, Recall and F-measure and it equals $95.1 \%, 76.5 \%$ and $84.4 \%$ respectively. While Naïve Bayes achieved best Precision and it equals to $85.1 \%$. As we know this lexicon is the first lexicon of Sudanese dialect. And according to the results, our work could be very valuable to identify positive and negative opinions of customers for marketing purpose. And also, for detect negative comments related delicate issues (racism, etc..).

\section{ACKNOWLEDGMENT}

We would like to express our families and friends in social media for their participation in the survey which supported our work in this way and helped us get results of better quality. Also, a special gratitude goes out to the Technical University of Madrid (UPM) for providing the funding for the publication.

\section{REFERENCES}

[1] Huda, J Abdelhameed, and Susana Muñoz - Hernández. "Emotion and opinion retrieval from social media in Arabic language: Survey." Information and Communication Technologies for Education and Training and International Conference on Computing in Arabic (ICCA-TICET), 2017 Joint International Conference on, pp. 1-8. IEEE, 2017.

[2] Duwairi, R. M., Nizar A. Ahmed, and Saleh Y. Al-Rifai." Detecting sentiment embedded in Arabic social media-A lexicon-based approach." Journal of Intelligent \& Fuzzy Systems 29.1: 107-117, 2015.

[3] Soliman, Taysir Hassan A., M. Ali M. Abdel Rahman Hedar, and M. M. Doss." Mining Social Network's Arabic Slang Comments." Proceedings of IADIS European Conference on Data Mining. Vol. 22, 2013.

[4] Pang, Bo, and Lillian Lee. "A sentimental education: Sentiment analysis using subjectivity summarization based on minimum cuts." Proceedings of the 42nd annual meeting on Association for Computational Linguistics. Association for Computational Linguistics, 2004.

[5] Melville, Prem, Wojciech Gryc, and Richard D. Lawrence. "Sentiment analysis of blogs by combining lexical knowledge with text classification." Proceedings of the 15th ACM SIGKDD international conference on Knowledge discovery and data mining. ACM, 2009.

[6] Wilson, Theresa, Janyce Wiebe, and Paul Hoffmann. "Recognizing contextual polarity: An exploration of features for phrase-level sentiment analysis." Computational linguistics 35.3: 399-433, 2009.

[7] Soliman, Taysir Hassan, M. A. Elmasry, A. Hedar, and M. M. Doss. "Sentiment analysis of Arabic slang comments on Facebook." International Journal of Computers \& Technology 12, no. 5: 3470-3478, 2014.

[8] Helmy, Tarek, and Abdirahman Daud. "Intelligent agent for information extraction from Arabic text without machine translation." Proceedings of the 1st International Workshop on Cross-Cultural and Cross-Lingual Aspects of the Semantic Web. Vol. 1. 2010. [C3LSW2010] Workshop on Cross-Cultural and Cross-Lingual Aspects of the Semantic Web Shanghai, China, 2010.

[9] El-Halees A., "Arabic Opinion Mining Using Combined Classification Approach." Proceedings of the International Arab Conference on Information Technology, ACIT, Naif Arab University for Security Science (NAUSS), (Riyadh, Saudi Arabia), 2011.

[10] Al-Kabi, Mohammed N., Amal H. Gigieh, Izzat M. Alsmadi, Heider A. Wahsheh, and Mohamad M. Haidar. "Opinion mining and analysis for Arabic language." International Journal of Advanced Computer Science and Applications (IJACSA), SAI Publisher 5, no. 5: 181-195, 2014.

[11] Al-Kabi, Mohammed N., Nawaf A. Abdulla, and Mahmoud Al-Ayoub. "An analytical study of Arabic sentiments: Maktoob case study." Internet
Technology and Secured Transactions (ICITST), 2013 8th International Conference for. IEEE, 2013.

[12] A. El-Halees, "Arabic Opinion Mining Using Combined Classification Approach." Proceedings of the International Arab Conference on Information Technology, Zarqa, Jordan, 2011.

[13] Al-Kabi, Mohammed, Amal Gigieh, Izzat Alsmadi, Heider Wahsheh, and Mohamad Haidar. "An opinion analysis tool for colloquial and standard Arabic". The Fourth International Conference on Information and Communication Systems (ICICS 2013), pp. 23-25, 2013.

[14] Soliman, T. H., Elmasry, M. A., Hedar, A., \& Doss, M. M. "Sentiment analysis of Arabic slang comments on Facebook." International Journal of Computers \& Technology, 12(5), 3470-3478, 2014.

[15] Nakov, Preslav , Sara Rosenthal, Svetlana Kiritchenko, Saif M. Mohammad, Zornitsa Kozareva, Alan Ritter, Veselin Stoyanov, and Xiaodan Zhu. "Developing a successful SemEval task in sentiment analysis of Twitter and other social media texts." Language Resources and Evaluation 50, no. 1: 35-65, 2016.

[16] Elhawary, Mohamed, and Mohamed Elfeky. "Mining Arabic business reviews." Data Mining Workshops (ICDMW) IEEE International Conference on. IEEE, 2010.

[17] Al-Subaihin, Afnan A., Hend S. Al-Khalifa, and AbdulMalik S. Al-Salman. "A proposed sentiment analysis tool

for modern Arabic using human-based computing." Proceedings of the 13th International Conference on Information integration and Web-based Applications and Ser-vices. ACM, 2011.

[18] Rastogi, S. S. K., Rohit Singhal, and Anil Kumar. "An Improved Sentiment Classification using Lexicon into SVM." International Journal of Computer Applications 95, no. 1 (2014).

[19] Lee, Lam Hong, et al. "A review of nearest neighbor-support vector machines hybrid classification models." Journal of Applied Sciences 10: 1841-1858 (2010).

[20]

https://medium.com/@sifium/machine-learningtypes-of-classification-9497bd4f2e14 5 May 2019 Time 11:30 am.

Huda Jamal Abdelhameed has a Master Degree in Computer Science by the Sudan University of Science and Technology (SUST) and graduated from Computer Science Department, College of Computer Science and Information Technology, Sudan University of Science and Technology.

She has been working as lecturer at the Computer Systems and Networks, Sudan University of Science and Technology since 2009. She coordinated the graduated projects in Sudan University of Science and Technology several times. She is part of the committees for discussion of graduation projects.

She was participated in many training courses which held in Sudan. She published a publication with title "Emotion and Opinion Retrieval from Social Media in Arabic" at Information and Communication Technologies for Education and Training and International Conference on Computing in Arabic (ICCA-TICET), 2017 Joint International Conference on, pp. 1-8. IEEE, 2017. She has a certificate from IEEE as an accredited reviewer.

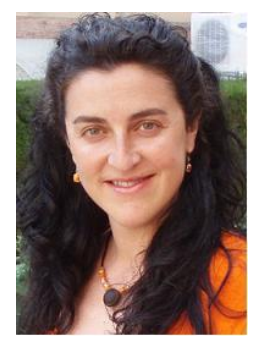

Susana Muñoz - Hernández

is $\mathrm{PhD}$ in Computer Science by the Technical University of Madrid (UPM), Master in Management of Information Technologies by the Ramón Llull University of Barcelona and graduate in International Affairs by the Society of International Studies of Madrid. She won the first prize in a national competition for talented young people organized by the University of $\mathrm{La}$ Salle of Madrid in 2003. She has professional experience in some companies (ICT and bank sector) and join research (national and European) projects of recognized prestige.

She has been working as associate professor at the Computer Science School of the Technical University of Madrid since 1998. She develops her research activity in the BABEL group (http://babel.ls.fi.upm.es) with more than eighty publications (http://babel.1s.fi.upm.es/ susana/publications.html). She is part of the committees of several international conferences in her area (ACM SAC, PROLE, IPMU, WCAFR, IWANN, SERVICE COMPUTATION, EDUCON, TALE, FCTA en IJCCI, WomENcourage, DEV, ECSEE, FUTURE COMPUTING). She coordinated the European Master in Computational Logic (first official Erasmus Mundus master in Computer 
Science) since 2004 till 2008. She was member of the Manager Committee of the Spanish Platform for Software and Services since 2008 till 2010

She set up the cooperation group TEDECO (Technology for the Development and the Cooperation, http://tedeco.fi.upm.es) in 2006 and she has been its director since then. She has directed various projects oriented to improve education in developing countries (Burundi, Ethiopia, Kenya, El Salvador, ...). She is director of the educational innovation group TIDE (Technology Innovation for the Educational Development, http://tide.fi.upm.es). Her teaching experience is in computer programming (methodology of programming, logic and functional languages, constraint programming, fuzzy logic, ...), free software applications and personal skills (communication, relation, presentations in public, negotiation, conflict solving, management of inter-cultural teams,...).

She is member of the advisory board of the direction of Cooperation for the Development of UPM International Relations and member of the advisory board of the OCUD (University Observatory of Cooperation for Development, http://www.ocud.es) that is part of the CRUE (Conference of Rectors of Spanish Universities). She received the 2011 UPM prize of cooperation for the development in international research. She is member of the Management Board of the itdUPM (Center of Innovation in Technology for the Human Development, http://www.itd.upm.es). 